\title{
LETTER
}

\section{Statistical correlations of an anyon liquid at low temperatures}

\author{
F. M. D. Pellegrino ${ }^{1}$, G. G. N. Angilella ${ }^{2 *}$ N. H. March ${ }^{3,4}$, R. Pucci ${ }^{2}$ \\ ${ }^{1}$ Scuola Superiore di Catania, Università di Catania, \\ Via S. Nullo, 5/i, I-95123 Catania, Italy, \\ and INFN, Sez. Catania, Via S. Sofia, 64, I-95123 Catania, Italy \\ ${ }^{2}$ Dipartimento di Fisica e Astronomia, Università di Catania, \\ and CNISM, UdR Catania, and INFN, Sez. Catania, \\ Via S. Sofia, 64, I-95123 Catania, Italy \\ ${ }^{3}$ Oxford University, Oxford, UK \\ ${ }^{4}$ Department of Physics, University of Antwerp, \\ Groenenborgerlaan 171, B-2020 Antwerp, Belgium
}

(Received on 8 February 2008, in final form)

\begin{abstract}
Using a proposed generalization of the pair distribution function for a gas of noninteracting particles obeying fractional exclusion statistics in arbitrary dimensionality, we derive the statistical correlations in the asymptotic limit of vanishing or low temperature. While Friedel-like oscillations are present in nearly all non-bosonic cases at $T=0$, they are characterized by exponential damping at low temperature. We discuss the dependence of these features on dimensionality and on the value of the statistical parameter $\alpha$.
\end{abstract}

Keywords: Electron liquid; Quantum Hall effect; Fractional statistics; Pair correlation function.

Running head: Low-temperature correlations of anyon liquids.

${ }^{*}$ Corresponding author. E-mail: giuseppe. angilella@ct.infn.it 
Fractional statistics has been introduced to describe the fractional quantum Hall effect in low-dimensional, correlated electron quantum liquids. [1, 2, 3, 4, 5, 6, The elementary excitations of such systems, usually dubbed anyons, can be characterized either through the phase factor $e^{i \alpha \pi}(0 \leq \alpha \leq 1)$ picked up by the overall wave-function upon exchange of two particles, of by the variation of size $\Delta D$ of the available Hilbert space corresponding to a variation $\Delta N$ of the number of particles, through the ratio $g=-\Delta D / \Delta N(0 \leq g \leq 1)$. The limiting cases $\alpha=0, g=0[\alpha=1, g=1]$ correspond to boson [fermion] statistics, respectively. While the former characterization, corresponding to exchange fractional statistics, applies to dimensions $d \leq 2$ only, the latter characterization, corresponding to exclusion statistics, does not suffer from such a restriction, and has been considered for arbitrary dimensionality. The relation between the two definitions of fractional statistics is not completely settled, to date [7].

In this context, it has been recently proposed that topological excitations such as vortex rings (anyonic loops) in the three-dimensional chiral spin liquids may obey fractional non-Abelian statistics [8]. For the sake of simplicity, and in order to avoid confusion with the pair correlation function to be defined below, in the following we shall use the symbol $\alpha$ for the statistical parameter in both contexts.

Recently, the statistical correlations of an ideal gas of particles obeying fractional exchange statistics have been addressed, in arbitrary dimensionality, as a function of density, temperature, and statistical parameter $\alpha$, partly analytically and partly numerically, through the study of the pair correlation function $g(\mathbf{r})[9]$. This function represents the probability of simultaneously finding two anyons at positions $\mathbf{r}$ and $\mathbf{r}=0$, respectively. A general result, applying to all non-bosonic values of the fractional parameter $(0<\alpha \leq 1)$, is that the pair correlation function is characterized by Friedel-like oscillations, which become more pronounced for increasing $\alpha$ or density, or for decreasing $T$. A somewhat related study [10] considered the two-particle kernel in the lowest Landau level of a quantum Hall system, but now in $d=2$ and within the context of exchange fractional statistics.

In this Letter, we study some asymptotic properties of the pair correlation function at zero temperature as a function of density.

In the formalism of second quantization, the pair correlation function introduced above can be defined as:

$$
g(\mathbf{r})=\frac{\left\langle\Psi^{\dagger}(\mathbf{r}) \Psi^{\dagger}(0) \Psi(0) \Psi(\mathbf{r})\right\rangle}{n(\mathbf{r}) n(0)}
$$

where $\Psi^{\dagger}(\mathbf{r})[\Psi(\mathbf{r})]$ is a creation [annihilation] quantum field operator at position $\mathbf{r}$, $n(\mathbf{r})=\left\langle\Psi^{\dagger}(\mathbf{r}) \Psi(\mathbf{r})\right\rangle$ is the single-particle probability density at position $\mathbf{r}$, and $\langle\ldots\rangle$ denotes a quantum statistical average associated with the equilibrium distribution of the identical particle assembly under analysis.

In Ref. 9, we introduce a particular generalization of the commutation relations 
for the $\Psi$-fields, interpolating between boson and fermion statistics for $0 \leq \alpha \leq 1$ and arbitrary dimensionality $d$. These are reminiscent of the graded commutation relations of Greenberg [11, weakly violating conventional quantum statistics.

In this way, the pair correlation function for a translationally system of anyons obeying exclusion statistics can be written as:

$$
\begin{aligned}
g(r) & =1+\cos (\alpha \pi) \frac{\left|\left\langle\Psi^{\dagger}(r) \Psi(0)\right\rangle\right|^{2}}{n^{2}(0)} \\
& =1+\cos (\alpha \pi)\left|\frac{\tilde{n}(r)}{\tilde{n}(0)}\right|^{2},
\end{aligned}
$$

where

$$
\tilde{n}(r)=\int \frac{d^{d} \mathbf{k}}{(2 \pi)^{d}} e^{-i \mathbf{k} \cdot \mathbf{r}} n(\mathbf{k})
$$

is the Fourier trasform in $d$ dimensions of the distribution function $n(\mathbf{k})$ for exclusion anyons at equilibrium. While alternative derivations of the latter have been proposed by Ouvry et al. [12, 13, as well as viable approximations within the chemical collision model by March et al. [14, 15, 16, in the following we shall use Wu's definition [17]:

$$
n(\mathbf{k})=\frac{1}{w(\zeta)+\alpha}
$$

where $\zeta=\zeta(\mathbf{k})$, and $w(\zeta)$ satisfies the functional relation:

$$
w^{\alpha}(1+w)^{1-\alpha}=\zeta .
$$

In this Letter we treat the case of an ideal gas of anyons obeying exclusion exclusion statistics, and for their dispersion relation we assume the usual one as for a non-relativistic free particle:

$$
\epsilon(\mathbf{k})=\frac{\hbar^{2} k^{2}}{2 m}
$$

At $T=0$ and for each non-zero value of the statistical parameter $\alpha(0<\alpha \leq 1)$, Wu's distribution function Eq. (44) becomes a step function, as in Fermi statistics. In particular, it assumes the following limiting form:

$$
n(\epsilon)=\left\{\begin{array}{rr}
1 / \alpha, & \epsilon \leq \epsilon_{f, \alpha} \\
0, & \epsilon>\epsilon_{f, \alpha}
\end{array}\right.
$$

where $\epsilon_{f, \alpha}$ is a generalized Fermi energy, that can be defined in arbitrary dimension as the highest level occupied by $N$ particles at $T=0$. It is appropriate at this point to remind that under the same conditions in the bosonic case $(\alpha=0)$ the distribution function behaves like a Dirac delta function, $n(\epsilon)=N \delta(\epsilon)$. 
Therefore, inserting Eq. (7) into Eq. (3) allows us to derive the analytical expression of the anyon pair correlation function in arbitrary dimension $d$ at $T=0$ as:

$$
g(r)=1+\cos (\alpha \pi) \Gamma\left(\frac{d}{2}\right)\left(\frac{2}{k_{f, \alpha} r}\right)^{d} J_{d / 2}^{2}\left(k_{f, \alpha} r\right)
$$

where $J_{d / 2}(z)$ is a Bessel function of first kind of order $d / 2$ [18]. $k_{f, \alpha}$ represents the wavevector implicitly defined by:

$$
C_{d}\left(\frac{k_{f, \alpha}}{2 \pi}\right)^{d}=\alpha \frac{N}{V}
$$

where $C_{d}=\frac{\pi^{d / 2}}{\Gamma(d / 2)}$ denotes the volume of the unit sphere in $d$ dimensions. In the fermion case $(\alpha=1), \hbar k_{f, \alpha}$ represents the Fermi momentum.

In order to compare the cases with different values of the statistical parameter $\alpha$, we write the pair correlation function as:

$$
g(y)=1+\cos (\alpha \pi) \Gamma\left(\frac{d}{2}\right) \frac{2^{d}}{\alpha y^{d}} J_{d / 2}^{2}\left(\alpha^{1 / d} y\right),
$$

where $y=k_{f} r$ is a dimensionless variable, with $k_{f}$ the Fermi wavevector, which agrees with Eq. (9) when $\alpha=1$. For $0<\alpha \leq 1, g(y)$ has the same functional form of the fermionic limit, the differences being the factor $\cos (\alpha \pi) / \alpha$ and the dependence of $k_{f, \alpha}$ on the statistical parameter $\alpha$.

Figure 1, on the left hand side, shows the pair correlation function $g(y)$ at $T=0$ in $d=2$ for $\alpha=0-1$. In the fermionic regime $\left(\frac{1}{2}<\alpha \leq 1\right)$, the pair correlation function exhibits a hole centred at $y=0$, whose depth decreases with decreasing $\alpha$ until it disappears at $\alpha=\frac{1}{2}$. For $0<\alpha<\frac{1}{2} g(y)$ shows a hill centred at $y=0$, whose height decreases with increasing $\alpha$. In the bosonic limit $(\alpha=0)$, the pair correlation function $g(y)$ is a constant, $g(y)=2$, which is a known result. Figure 1, on the right hand side, allows us to appreciate the damped Friedel-like oscillations of the pair correlation function for each non-zero value of the statistical parameter $\alpha$, except for the case $\alpha=\frac{1}{2}$.

Making use of Eq. (8) and of the asymptotic properties of the Bessel function $J_{\nu}(z)$, in the limit $z \gg 1$, the wavelength $R$ of the Friedel-like oscillations in the pair correlation function can be derived as:

$$
R=\frac{2 \pi}{k_{f, \alpha}}=\sqrt{\pi}\left(\alpha \Gamma(d / 2) \frac{N}{V}\right)^{-\frac{1}{d}} .
$$

For each dimensionality $d$, at $T=0$ and at fixed density $N / V$, the wavelength $R$ depends explicitly on the statistical parameter $\alpha$. Indeed, $R$ increases with decreasing $\alpha$, and in the bosonic limit $(\alpha=0)$ the wavelength becomes infinity $(R=\infty)$. 

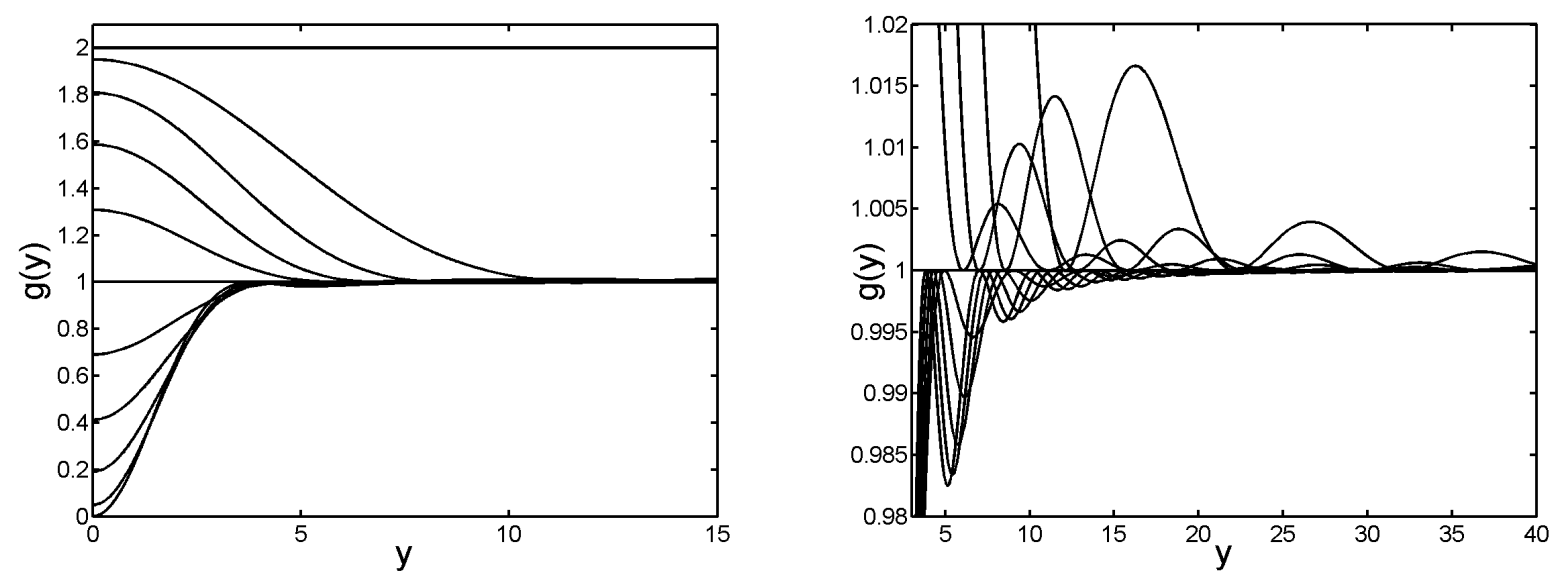

Figure 1: (Left) Pair correlation function $g(y)$ (in scaled units), Eq. (10), in $d=2$ for $\alpha=0-1$ ( $\alpha$ increases from top to bottom). (Right) The figure shows Friedel-like oscillations of the pair correlation function, which are present in each case with $\alpha \neq 0, \frac{1}{2}$.

On the basis of Eq. (11), one would find a finite wavelength $R$ also for $\alpha=\frac{1}{2}$. Therefore, the absence of Friedel-like oscillations in the pair correlation function (cf. Fig. 1, right panel, for $T=0$, and Ref. [9] for finite temperature) can only be related to the presence of the vanishing factor $\cos (\alpha \pi)$ in the original definition of the pair correlation function, Eq. (11). Indeed, in this case the factor $\cos (\alpha \pi)$ cancels every deviation of $g(\mathbf{r})$ from unity. In other words, the absence of Friedel-like oscillations in the pair correlation function in the so-called semionic case $\left(\alpha=\frac{1}{2}\right)$ is not a consequence of the exclusion statistics in momentum space, but of the exchange properties implied in our ad hoc generalization of the commutation relations for anyons obeying exclusion statistics 9]. Qualitatively similar results (not shown here) are obtained in $d=1$ and $d=3$.

Generally, Friedel oscillations in the many-body properties of fermion assemblies arise from the presence of a discontinuity in the Fermi distribution function at the Fermi level. This behaviour is characteristic of Wu's distribution function when $\alpha$ is non-zero. In the literature, it is known that at low but finite temperature the Friedel oscillations in the charge density around a screened impurity are exponentially damped with a characteristic decay length $\lambda^{2} k_{f}$, where $\lambda=\hbar \sqrt{2 \pi /\left(m k_{B} T\right)}$ is the thermal wavelength. Since at low temperature the behaviour of Wu's distribution function for non-zero $\alpha$ is equivalent to the Fermi-Dirac distribution, we find that the Friedel oscillations are exponentially damped with a characteristic decay length $\lambda^{2} k_{f, \alpha}$.

Making use of these remarks, we can derive an approximate form of the pair correla- 

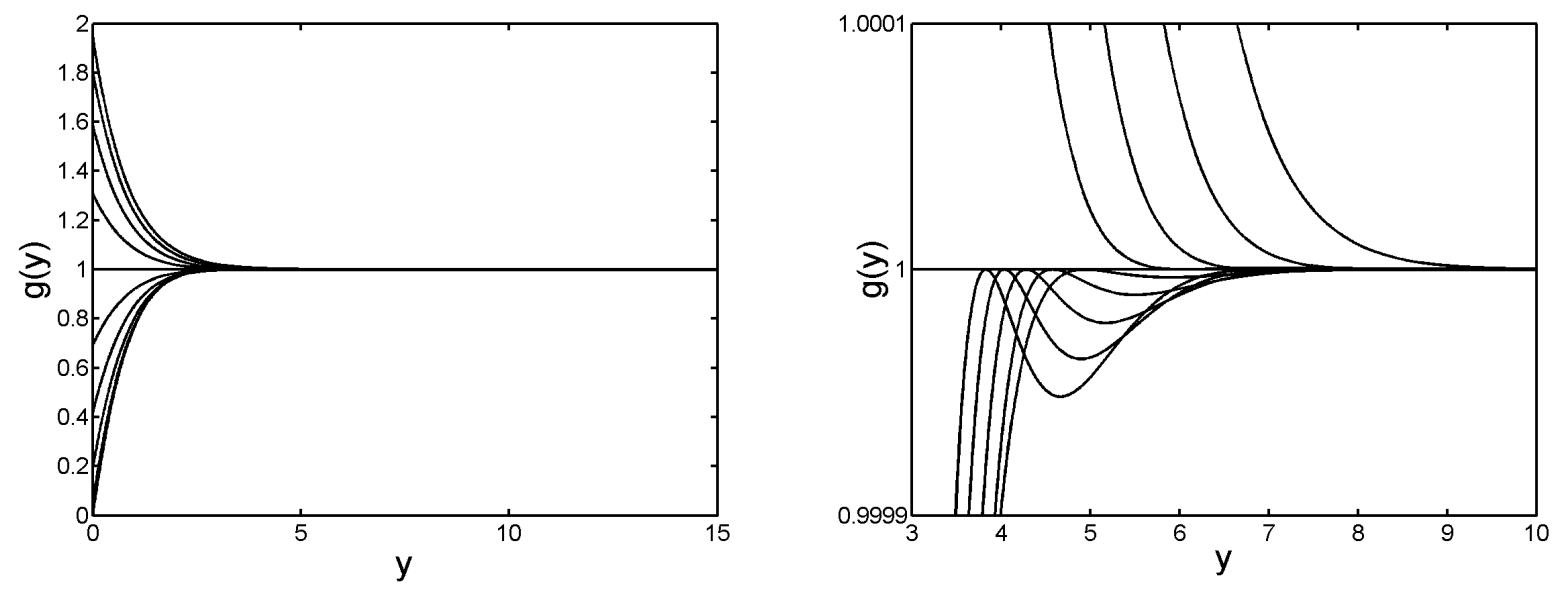

Figure 2: (Left) Approximate pair correlation function $g(y)$ (in scaled units), Eq. (10), in $d=2$ and with $\lambda^{d} N / V=1 / 15$, for $0<\alpha \leq 1$ ( $\alpha$ increases from top to bottom). (Right) Showing Friedel-like oscillations in $g(y)$.

tion function at finite temperature. The idea consists in weakly modifying the function $g(r)$, Eq. (2), which is exact at $T=0$. We multiply it by a damping factor which has a characteristic decay length:

$$
L=\lambda^{2} k_{f, \alpha} .
$$

In this way the approximate correlation function can be defined as:

$$
g(r)=1+\exp \left(-\frac{r}{L}\right) \cos (\alpha \pi) \Gamma\left(\frac{d}{2}\right)\left(\frac{2}{k_{f, \alpha} r}\right)^{d} J_{d / 2}^{2}\left(k_{f, \alpha} r\right) .
$$

Analogously, at $T=0$ we can rewrite the pair correlation function by means of a change of variable, and we obtain:

$$
g(y)=1+\exp \left(-\frac{y}{Y}\right) \cos (\alpha \pi) \Gamma\left(\frac{d}{2}\right) \frac{2^{d}}{\alpha y^{d}} J_{d / 2}^{2}\left(\alpha^{1 / d} y\right)
$$

where the dimensionless quantity $Y$ acts as a characteristic decay length, and is defined as:

$$
Y=\alpha^{\frac{1}{d}} \lambda^{2} k_{f}^{2} .
$$

Figure 2 shows the approximate pair correlation function in $d=2$ for $\lambda^{d} N / V=1 / 15$. This value of the scaled density is still far from the classical limit, since the average distance between two particles is comparable to the thermal wavelength. Likewise, the 
approximate pair correlation function at $T=0$ shows a hill centred at $y=0$ when the statistical parameter $\alpha$ is close to the bosonic limit, $0 \leq \alpha<\frac{1}{2}$, and shows instead a hole near the fermionic limit, $\frac{1}{2}<\alpha \leq 1$.

Moroever, Figure 2 shows that the oscillations are absent only for $\alpha=0$ and $\frac{1}{2}$. For the cases which are close to the bosonic limit $\left(0 \leq \alpha<\frac{1}{2}\right)$ the damping term extinguishes completely the Friedel-like oscillations. On the other hand, for the cases which are close to the fermionic limit $\left(\frac{1}{2}<\alpha \leq 1\right)$, under the same conditions, the oscillations suffer from a heavy attenuation but are still visible. It is clear from Eq. (15) that under the same conditions (i.e. temperature, density, dimensionality), the damping of the Friedel-like oscillations depends explicitly on the parameter $\alpha$. Indeed, the value of the scaling variable $Y$ increases with increasing $\alpha$. Near the bosonic limit, the characteristic decay length is so short that the oscillations are completely damped out, whereas close to the fermionic limit the characteristic decay length is so large that the first Friedellike oscillations survive. Pictorially, we can state that the ideal anyonic liquids losing Friedel-like oscillations in their pair correlation function at low $T$, are those which have smaller volume within the generalized Fermi surface.

Summarizing, following a proposed generalization of the pair correlation function $g(\mathbf{r})$ for a gas of non-interacting anyons obeying exclusion fractional statistics in arbitrary dimensions, we have studied the asymptotic statistical correlations between anyons at $T=0$ and in the limit of low temperature. We find Friedel-like oscillations in $g(\mathbf{r})$ in all quasi-fermionic cases at $T=0$, except for the value $\alpha=\frac{1}{2}$ of the statistical parameter. At low temperature, such oscillations are damped exponentially, with a characteristic decay length which increases with decreasing density and increasing dimensionality.

\section{Acknowledgements}

This paper was brought to completion during a visit of GGNA and NHM to the Department of Physics, University of Antwerp. Thanks are due to Professors C. Van Alsenoy and D. Lamoen for their warm hospitality. NHM was partially supported by FWOVlaanderen under Project No. G.0425.05.

\section{References}

[1] J. M. Leinaas and J. Myrheim (1977). Nuovo Cimento B, 37, 1.

[2] F. Wilczek (1982). Phys. Rev. Lett., 48, 1144.

[3] F. Wilczek (1982). Phys. Rev. Lett., 49, 957.

[4] R. B. Laughlin (1983). Phys. Rev. Lett., 50, 1395.

[5] R. B. Laughlin (1983). Phys. Rev. B, 27, 3383. 
[6] R. B. Laughlin (1988). Phys. Rev. Lett., 60, 2677. [61, 379(E) (1988)].

[7] A. D. Speliotopoulos (1997). J. Phys. A: Math. Gen., 30, 6177.

[8] T. Si and Y. Yu (2007). ..., ..., ... Preprint arXiv:0712.4231.

[9] F. M. D. Pellegrino, G. G. N. Angilella, N. H. March and R. Pucci (2007). Phys. Rev. E, 76, 061123.

[10] D. Sen, M. Stone and S. Vishveshwara (2007). ...,...,... Preprint arXiv: 0712.0879.

[11] O. W. Greenberg (1991). Phys. Rev. D, 43, 4111.

[12] A. Dasnières de Veigy and S. Ouvry (1994). Phys. Rev. Lett., 72, 600.

[13] A. Dasnières de Veigy and S. Ouvry (1995). Mod. Phys. Lett. B, 9, 271.

[14] N. H. March, N. Gidopoulos, A. K. Theophilou, M. J. Lea and W. Sung (1993). Phys. Chem. Liq., 26, 135.

[15] N. H. March (1993). J. Phys.: Condens. Matter, 5, B149.

[16] N. H. March (1997). Phys. Chem. Liq., 34, 61.

[17] Y.-S. Wu and Y. Yu (1995). Phys. Rev. Lett., 75, 890.

[18] I. S. Gradshteyn and I. M. Ryzhik, Table of Integrals, Series, and Products (Academic Press, Boston, 1994), 5th edition. 